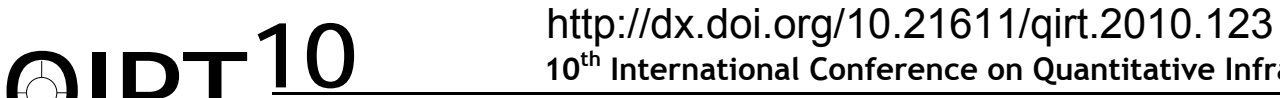 \\ $10^{\text {th }}$ International Conference on Quantitative InfraRed Thermography \\ July 27-30, 2010, Québec (Canada)
}

\section{Wavelet based statistical descriptor for precise decision making in the interpretation of breast cancer thermographs}

\author{
N.Selvarasu* and Alamelu Nachiappan**
}

\begin{abstract}
*Research Scholar, Sathyabama University, Jeppiaar Nagar, Rajiv Gandhi Road, Chennai 600119 onsselvan123@yahoo.co.in, onsselvan123@gmail.com

**Dept. of Electrical and Electronics Engg., Pondicherry Engineering College, Pondicherry, nalam67@yahoo.com
\end{abstract}

\begin{abstract}
Early detection of breast cancer can considerably reduce the mortality rate. Infrared thermography is the best suited sensor for capturing cancer at an early stage. Infrared camera captures the temperature variations and maps them into thermographs. However interpretation of these thermographs is complex as temperature variation is subjective in nature. This paper aims at determining suitable statistical parameters for automatic interpretation of cancer regions.
\end{abstract}

\section{Introduction}

To reduce the mortality rate, it is necessary to detect breast cancer at an early rate. Infrared Thermography [1-2], a noninvasive, non-contact medical imaging technique widely accepted for early detection of breast cancer maps temperature into thermographs. These thermographs must be analyzed for cancer detection. For a normal person, thermographs are symmetrical and uniform. If there is an asymmetry then it indicates an abnormality namely breast cancer or fibroadenoma. Hence the nature of the abnormality can be assessed by quantifying the hotspots. Abnormality analysis techniques are complicated because generalized extraction can not be made due to subjective temperature variation in temperature. This paper proposes a suitable wavelet descriptor for describing the abnormality region.

\section{Methodology}

The acquired color thermograph is converted into gray scale [3]. The thermograph is then divided vertically into two sections to separate left and right breasts of nearly equal size (column-wise). Mean intensity is determined for both the sections. If there is a significant variation in mean then it represents an abnormality. If an abnormality is present then these sub images are further divided into upper and lower subsections else the process ends in this step itself. Discrete Wavelet transform is applied on these subsections using Daubechies wavelets of order 2, 4, 6 and 8. Single level decomposition is done. Mean is calculated for approximation, horizontal detail, vertical detail and diagonal detail of left and right subsections and are compared.

\section{Results and Discussion}

A breast thermograph containing cancer region is considered. The image is divided into left and right sections. The mean in spatial domain for left and right section intensities are 131.7863 and 118.8641 pixels respectively. It is found that there is a significant variation in intensity and hence these images are further divided into upper and lower subsections. The mean in spatial domain for upper and lower subsections for left and right subsections are 137.1444, 126.4282, 130.6816 and 107.0466 pixels respectively. It is found that cancer region is found in right lower subsection. Discrete wavelet transform is applied using db2, db4, db6 and db8. Mean for approximation, horizontal, vertical and detailed coefficients for db2, db4, db6 and db8 wavelets are as shown in Table 1.

Table 1: Mean for approximation, horizontal, vertical and detailed coefficients for $d b 2, d b 4, d b 6$ and db8 wavelets for left and right lower subsections of breast cancer thermograph

\begin{tabular}{|c|c|c|c|c|c|}
\hline & & Approximation & Horizontal Detail & Vertical detail & Diagonal detail \\
\hline \multirow[b]{2}{*}{ Db2 } & left & 0.9844 & $-3.1306 e-004$ & $3.2990 \mathrm{e}-004$ & $1.7449 \mathrm{e}-004$ \\
\hline & right & 0.8311 & $2.8288 \mathrm{e}-004$ & -0.0036 & $-2.7886 e-004$ \\
\hline \multirow[b]{2}{*}{ Db4 } & left & 0.9730 & $-1.5925 e-004$ & $3.4364 \mathrm{e}-004$ & $1.9657 \mathrm{e}-004$ \\
\hline & right & 0.8331 & $3.4521 \mathrm{e}-004$ & -0.0014 & $-1.9054 e-004$ \\
\hline \multirow[b]{2}{*}{ Db6 } & left & 0.9651 & $-3.3478 e-006$ & $2.8318 \mathrm{e}-004$ & $1.7937 e-004$ \\
\hline & right & 0.8340 & $3.8262 \mathrm{e}-004$ & $-4.4236 e-004$ & $-2.0267 e-004$ \\
\hline
\end{tabular}




\begin{tabular}{|c|l|c|c|r|r|}
\hline \multirow{2}{*}{ Db8 } & Left & 0.9597 & $1.4137 \mathrm{e}-005$ & $1.8585 \mathrm{e}-004$ & $1.2216 \mathrm{e}-004$ \\
\cline { 2 - 6 } & right & 0.8356 & $4.4019 \mathrm{e}-004$ & $1.8306 \mathrm{e}-004$ & $-1.5656 \mathrm{e}-004$ \\
\hline
\end{tabular}

The corresponding mean for left and right subsections for approximation, horizontal details, vertical details and diagonal details are shown in Figure1-4.

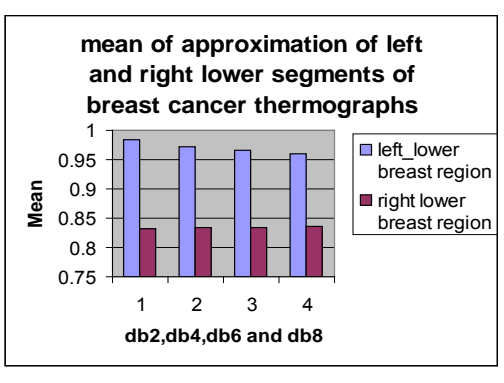

Fig.1: mean for Approximation co-efficients

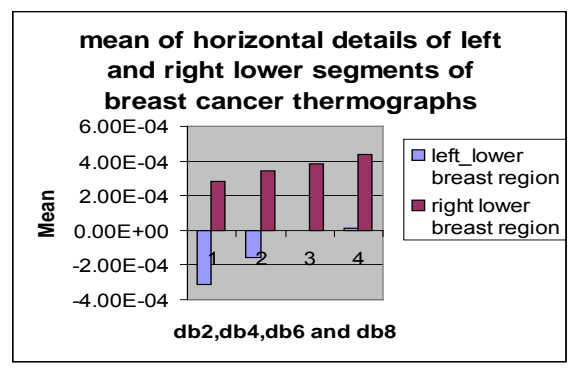

Fig.2: mean for horizontal detail

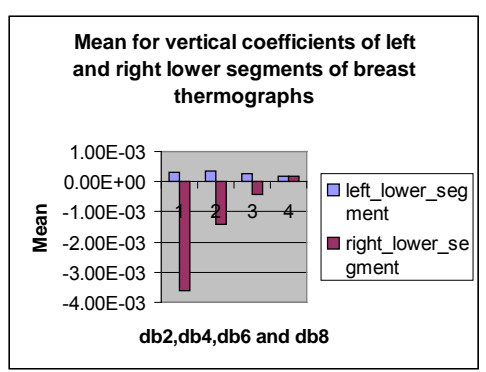

Fig.3: mean for vertical detail

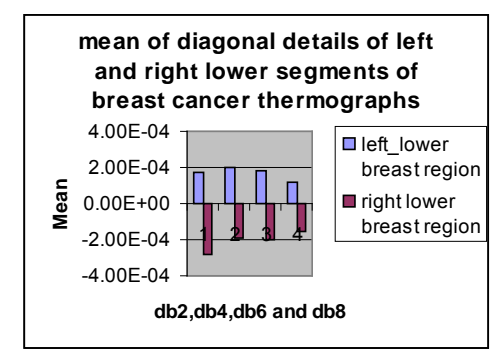

Fig.4: mean for diagonal details

It is found that for all the orders of wavelet, same variations are seen in approximation co-efficients between left and right subsections. But approximation co-efficients are not reliable for it can not show the variations as it is filtered by low pass filter co-efficients both row-wise and column-wise. Hence it is necessary to analyze the detailed co-efficients. Abnormality can be best described when there is abrupt variation in mean between abnormal section and the corresponding normal section. From the graphs it is found that though db6 and db8 provide better results for horizontal detail, they do not show maximum variations for other co-efficients. But $\mathrm{db} 2$ and $\mathrm{db} 4$ provide significant change in mean for all the detailed co-efficients.

\section{Conclusion}

Assessment of abnormality can be done by quantitatitive characterization of hotspots. Statistical parameters can best describe hotspot. Single level decomposition is done and it is found that $\mathrm{db} 2$ and $\mathrm{db} 4$ provide better results for all the three co-efficients namely horizontal, vertical and diagonal details. Hence statistical parameters can be measured for db2 and db4 decomposed images and can be further used for decision making. In this work comparison is based on mean only. The work can be extended for other statistical parameters also.

\section{REFERENCES}

[1]. Maldague X., Nondestructive testing handbook volume 3: Infrared and Thermal Testing, American Society for Nondestructive Testing, 2001.

[2]. Maldague X., Theory and Practice of Infrared Technology for Non Destructive Testing, John-Wiley \& Sons, 2001.

[3]. Rafael C. Gonzalez, Richard E. Woods, "Digital Image Processing”, Prentice Hall of India, 2005 